\title{
Minitracheotomy in the Early Respiratory Management of Patients with Spinal Injuries
}

\author{
A. Gupta, MS Orth, DRM, FRCS, ${ }^{1}$ M. R. McClelland, BSc, FRCS, ${ }^{2}$ A. \\ Evans, MCSP, ONC, ${ }^{3}$ W. S. El Masri, FRCS ${ }^{4}$ \\ ${ }^{1}$ Orthopaedic Registrar, Univeristy Department of Orthopaedics, Royal Liverpool \\ Hospital, Liverpool, UK, ${ }^{2}$ Senior Registrar in Spinal Injuries, Midland Spinal \\ Injuries Centre, Oswestry, UK, ${ }^{3}$ Senior Physiotherapist, Midland Spinal Injuries \\ Centre, Oswestry, UK, ${ }^{4}$ Consultant Surgeon in Spinal Injuries/Medical Director, \\ Midland Spinal Injuries Centre, Oswestry, UK.
}

\section{Summary}

Minitracheotomy is a new technique of tracheal suction by the introduction under local anaesthetic of a small bore tube into the trachea through the cricothyroid membrane. The use of minitracheotomy in the early management of respiratory problems in patients with spinal injuries is described with a few illustrative cases. This technique is an adjunct to good physiotherapy in clearing secretions from the trachea. Its advantages and disadvantages are discussed.

Key words: Spinal cord injury; Respiratory problems; Tracheostomy; Minitracheotomy.

The majority of early deaths of patients with spinal cord injuries are due to respiratory complications (Tribe, 1963; Cheshire, 1964; Cheshire and Foster, 1964; Cheshire, 1966; Silver and Gibbon, 1968; Bellamy et al., 1973; Kiwerski et al., 1981; Carter, 1987). Carter stated that nearly $70 \%$ of all spinal injuries in his review might be expected to have a marked predisposition for respiratory problems. Most of these problems arise due to an inability of the patient to clear the increased secretions from the mucous glands and transudation of fluid into the alveoli (Kierwski et al., 1981). Tetraplegic patients have a reduced vital capacity due to decreased inspiratory capacity and low expiratory reserve volume (Forner, 1968) and are predisposed to developing inspiratory muscle fatigue (Gross et al., 1980). They are also, unable to make use of their abdominal muscles to cough. Silver and Gibbon (1968) emphasised that the greatest single cause of both death and long term morbidity in complete tetraplegia was respiratory infection as a result of retained secretions.

Respiratory failure can often be avoided by careful monitoring of the vital signs, including respiratory rate, vital capacity and abdominal girth, along with physiotherapy from the time of admission. The problem of retained secretions requires aggressive management with the use of antibiotics, nebulised beta-2stimulators and vigorous physiotherapy.

Many patients are intubated or tracheotomised and ventilated in an attempt 
to assist gas exchange and help clear secretions (Bellamy et al., 1973; Gardner et al., 1986). Unfortunately, tracheostomy has its own complications (Cheshire, 1964) which do not appear to have been reduced by the use of modern plastics and low pressure cuffs (Hsu et al., 1987). The most serious complication is laryngotracheal stenosis which was reported by $\mathrm{Hsu}$ et al. to have occurred in $13 \%$ of their patients requiring tracheostomy.

Various methods have been tried to clear the major airways of secretions including fibre optic laryngoscopy (Cheshire and Coats, 1965) and modified Magill's tube for endobronchial suction (Cheshire and Foster, 1964).

Matthews and Hopkinson (1984) described a new technique called minitracheotomy in which a small canula ( $4 \mathrm{~mm}$ diameter) is introduced into the trachea through the cricothyroid membrane under local anaesthetic. The canula facilitates suction of the secretions in the trachea without exhausting either the patient or the physiotherapist. Preston et al. (1986) described its use in 4 patients. They were able to avoid bronchoscopy, intubation and ventilation in these patients using this technique.

In the present study we describe the use of minitracheotomy in 8 patients with spinal injury and discuss the role and indications of minitracheotomy in relation to this form of injury.

\section{Materials and methods}

In the years 1986 and 1987 a total of 131 acute traumatic spinal injuries were admitted to the Midland Spinal Injuries Centre, of which over 50\% arrived within 48 hours of injury. Seventy four $(57 \%)$ had cervical spine injuries. Minitracheotomy has been used in the management of 8 of these patients and they fall into two groups.

Group 1 patients $(1,2,3,4$ and 5$)$ had minitracheotomy as a primary procedure, whereas in group 2 patients $(6,7$ and 8$)$ a minitracheotomy tube was used as a method of tracheal suction whilst weaning patients off their formal tracheostomy.

Primary insertion of a minitracheotomy tube is a straightforward procedure which can be carried out on the ward. Care must, of course, be exercised in handling the patients neck especially in those with hyperextension injuries and this may be a relative contraindication to the procedure as some extension of the neck may be needed. The contents of the sterile pack are seen in Figure 1. Insertion involves palpating the cricothyroid membrane in the midline and infiltrating the overlying skin and tissues down to the membrane with local anaesthetic. A stab incision is then made through the skin and membrane with the guarded scalpel provided and the flexible trochar inserted through this wound into the trachea. The minitracheotomy tube is then fed over the trochar into the trachea and the trochar is removed. After tying the tube in place with a small dressing around it, suction is carried out with a fine-bore catheter to remove any blood and secretions from the airway.

In group 2 patients, the standard plastic or silver tracheostomy tubes were simply replaced by the minitracheotomy tube and a clean dressing applied.

\section{Case report 1}

A 56-year-old man, was transferred to the Midland Spinal Injuries Centre (MSIC) from a neighbouring hospital. On admission he was found to have a C5 complete 


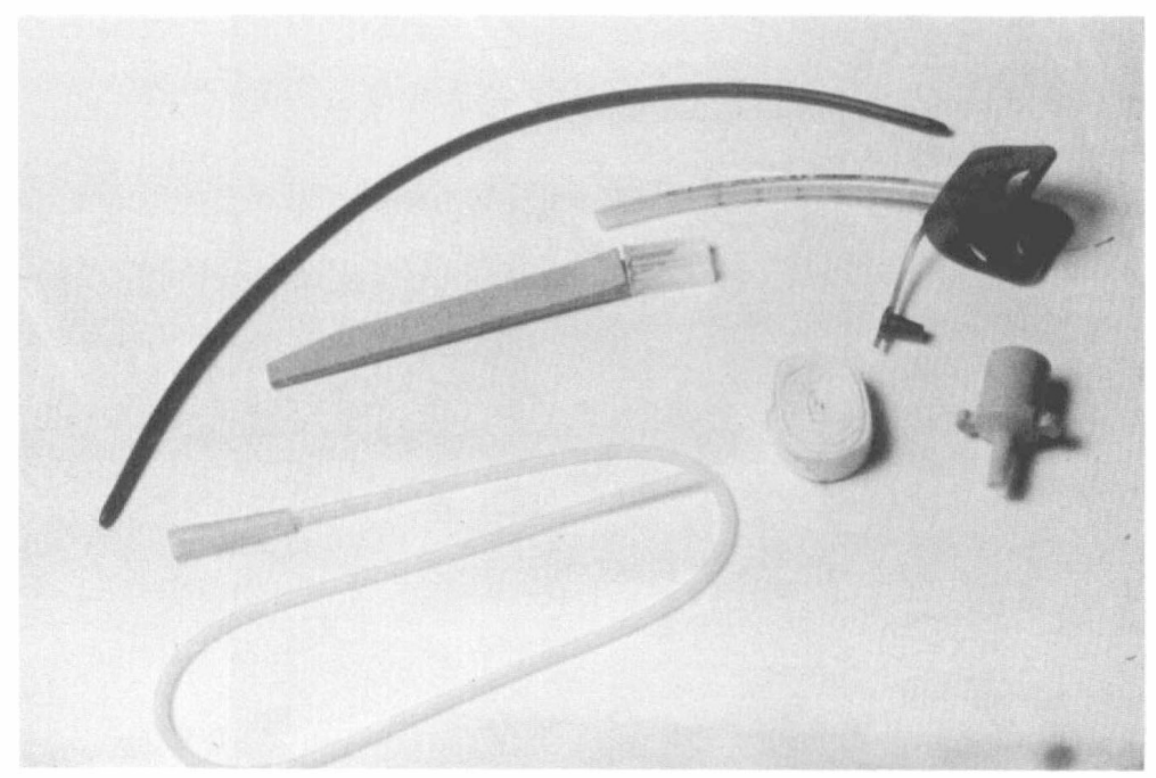

Figure 1 The contents of a minitracheotomy set.

tetraplegic due to $\mathrm{C} 4$ on $\mathrm{C} 5$ bilateral facet dislocation. He had a past history of chest tuberculosis as well as chronic bronchitis. Moreover, he was a very heavy smoker. His respiratory function was further compromised by abdominal distention from a paralytic ileus. He was expectorating thick purulent sputum and had widespread expiratory rhonchi in his chest. His vital capacity was $800 \mathrm{cc}$ and he had a $\mathrm{PaO}_{2}$ of $9.0 \mathrm{kP}\left(\mathrm{PaCO}_{2} 3.5\right.$ $\mathrm{kP}$ ) on $24 \%$ oxygen. Chest X-ray showed bilateral patchy consolidation with evidence of old calcified TB.

Initially, he was treated with vigorous physiotherapy, oxygen inhalation, intravenous and nebulised salbutamol and antibiotics. Two days later his condition deteriorated, he became tachypnoeic and was unable to co-operate with assisted coughing. There were crepitations throughout his lung fields but he was able to cough up only small amounts of purulent sputum. His vital capacity was between 600 and $800 \mathrm{ml}$ and his $\mathrm{PaO}_{2}$ had dropped to $6.5 \mathrm{kP}\left(\mathrm{PaCO}_{2} 3.9 \mathrm{kP}\right)$ on $28 \%$ oxygen. A minitracheotomy was performed and large amounts of secretions were removed with a marked improvement in his condition. Continued daily suction with humidification and vigorous physiotherapy improved his condition and his $\mathrm{PaO}_{2}$ rose to $8.4 \mathrm{kP}$ on $28 \%$ oxygen. He continued to require regular suction through the minitracheotomy tube for several weeks. It was removed at 60 days by which time he could expectorate normally with assistance and the tube was no longer required.

\section{Case report 2}

A 47 -year-old man was transferred to the MSIC 48 hours following a road accident in which he had been made tetraplegic at the level of $\mathrm{C} 5$. On arrival he had reduced air entry in the right side of his chest and his $\mathrm{PaO}_{2}$ was $4.4 \mathrm{kP}\left(\mathrm{PaCO}_{2} 4.7 \mathrm{kP}\right)$ on $28 \%$ oxygen and his vital capacity was $700 \mathrm{ml}$. Chest X-ray showed a complete collapse of the right upper lobe. Vigorous chest physiotherapy was given and a repeat chest $\mathrm{X}$-ray showed re-expansion of the right lung. $\mathrm{His} \mathrm{PaO}_{2}$ rose to $7 \cdot 1 \mathrm{kP}$ and his vital capacity to $850 \mathrm{ml}$. However, despite continuous physiotherapy throughout that night and the following day, he had a further collapse of his right upper lobe. By now, the patient was 


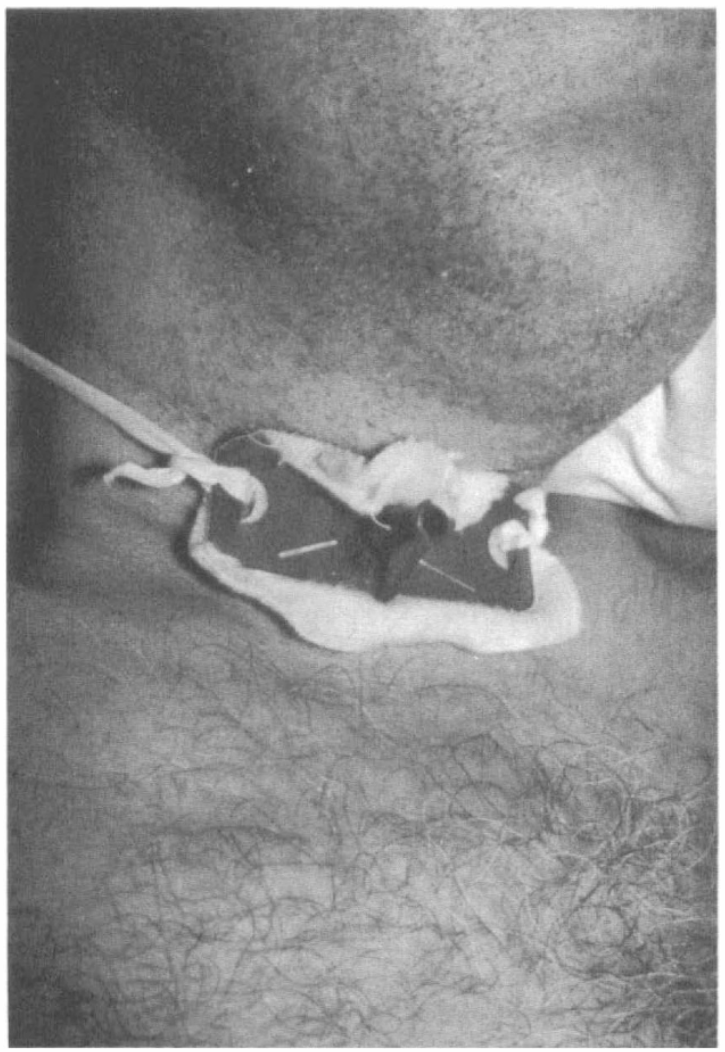

Figure 2 Minitracheotomy tube in-situ.

agitated, exhausted and unable to cough out the secretions. His $\mathrm{PaO}_{2}$ had dropped to $6.0 \mathrm{kP}\left(\mathrm{PaCO}_{2} 4.9 \mathrm{kP}\right)$ on $28 \%$ oxygen. Minitracheotomy was performed and copious amounts of secretions and mucous plugs were aspirated. Figure 2 shows the tube in situ. The right lung promptly re-expanded and the $\mathrm{PaO}_{2}$ rose to $8.8 \mathrm{kP}$ on $24 \%$ oxygen. Figures $3 \mathrm{~A}$ and $3 \mathrm{~B}$ are immediately pre- and post-minitracheotomy. He continued to produce large quantities of secretions which were sucked out through the minitracheotomy tube. It was some time before he was able to expectorate well enough with assistance and at this stage the minitracheotomy tube was left spigotted and finally withdrawn 52 days after insertion.

\section{Case report 3}

A 20-year-old was admitted to a local hospital with a depressed skull fracture, multiple right rib fractures with a right haemopneumothorax, wedge fracture of D6 and fracture dislocation of D10 with paraplegia below D10.

The initial management consisted of blood transfusion and insertion of a right intercostal drain and CVP line. The following day he was transferred to the MSIC. Chest physiotherapy was made difficult by pain from the fractured ribs and chest drain. Four days later the patient became tachypnoeic, tachycardic and cyanosed with a $\mathrm{PaO}_{2}$ of 4.9 $\mathrm{kP}$ on $28 \%$ oxygen and a vital capacity of $500 \mathrm{ml}$. Chest X-ray showed a collapse of the right middle and lower lobes. Minitracheotomy was performed and copious amounts of tenacious sputum removed with resultant re-inflation of his lung and a rise in $\mathrm{PaO}_{2}$ to $8.8 \mathrm{kP}$ on room air. The minitracheotomy tube was removed 2 weeks later without further complications. 


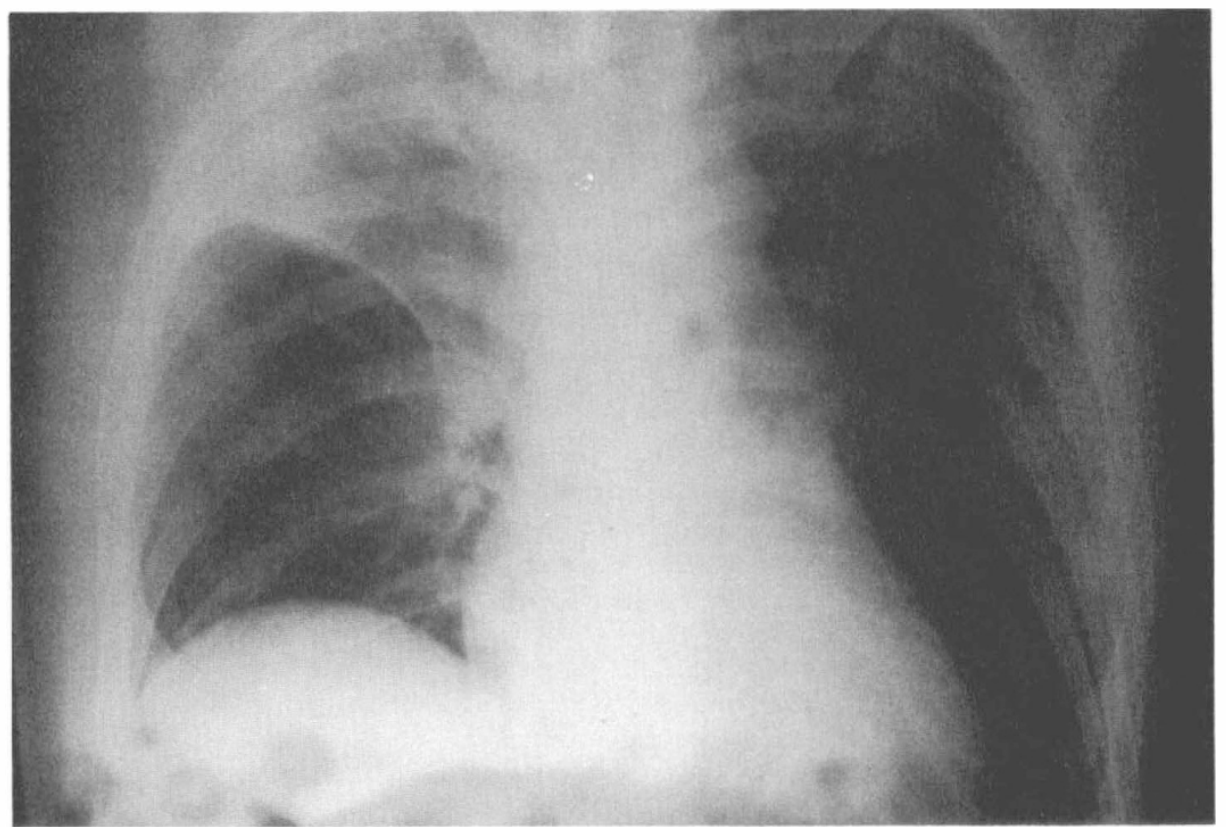

Figure 3A Chest X-ray prior to insertion of minitracheotomy tube showing right upper lobe collapse.

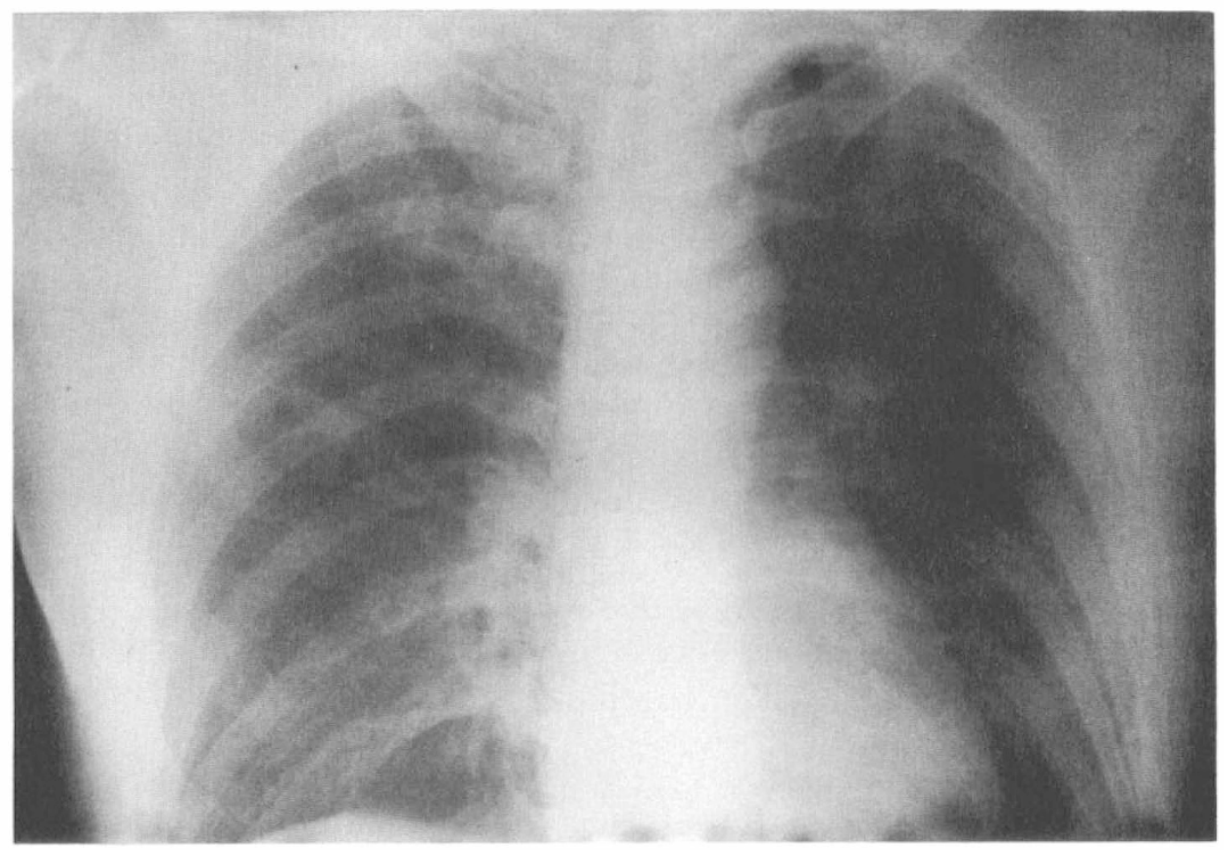

Figure 3B A repeat chest X-ray immediately following minitracheotomy with physiotherapy and suction. 


\section{Case report 4}

A 20-year-old motorcyclist sustained a head injury and a burst fracture of D9 with complete paraplegia. He was transferred to the MSIC within 48 hours but had been overinfused and upon arrival was confused and tachypnoeic. Chest X-ray showed a haemothorax which required bilateral chest drains. He was moved the same day to the ITU where he was intubated and ventilated. Over the following 10 days he was very ill with repeated collapse of his left lung. At the time of his extubation, a minitracheotomy tube was inserted to remove secretions. This remained in place until the patient was able to cough adequately and removed after 5 days.

\section{Case report 5}

A patient was admitted to hospital with a D1 fracture, a right brachial plexus lesion, fractured ribs, a right haemothorax and a compound skull fracture. When transfered to the MSIC several days later with a chest drain in situ he had reduced air entry in the left lung base and $\mathrm{PaO}_{2}$ of $9.8 \mathrm{kP}$ on oxygen. The following day he became tachypnoeic and his $\mathrm{PaO}_{2}$ dropped to $6.8 \mathrm{kP}$ on oxygen.

Chest X-ray showed collapse of the right lung with a mucus plug in the right main bronchus. There was no improvement after vigorous physiotherapy. A minitracheotomy tube was inserted and the secretions aspirated. $\mathrm{His} \mathrm{PaO}_{2}$ rose to $7.9 \mathrm{kP}$ on $24 \%$ oxygen and by the next morning his right lung was reinflated. The tube was removed 24 days later.

\section{Case report 6}

An 18-year-old motorcyclist was admitted, moribund, to an ITU after being involved in an accident. He was found to have a complete $\mathrm{C} 5$ tetraplegia as a result of a $\mathrm{C} 5$ fracture. $\mathrm{He}$ also had a fracture of the pedicle of $\mathrm{C} 2$. He was immediately intubated and ventilated and subsequently underwent splenectomy and insertion of intercostal drains for bilateral pneumothorax. The following day an elective tracheostomy was performed and ventilation continued through this. He was gradually weaned off the ventilator and his tracheostomy tube exchanged for a minitracheotomy tube to allow continued tracheal suction. This was removed 60 days post-injury.

\section{Case report 7}

A 15 -year-old boy, was admitted to a local hospital after a road traffic accident. He was noted to have fractures of $\mathrm{C} 1,5,6$ and 7 with a complete $\mathrm{C} 6$ tetraplegia. He was not immediately ventilated but when his vital capacity dropped to $350 \mathrm{ccs}$, with deterioration in his blood gases, a naso-endotracheal tube was inserted through which he was ventilated. He was transferred to the MSIC by helicopter 4 days after his injury. On arrival, he was found to have a complete collapse of his right lung due to blockage of the right main bronchus by the endotracheal tube. This problem was easily rectified and later a formal tracheostomy was carried out. Over the following days he was weaned off the ventilator and the portex tracheostomy tube was exchanged for a silver tube. This in turn, was changed to a minitracheotomy tube to allow tracheal suction to be continued and removed 101 days after injury.

\section{Case report 8}

A 24-year-old man was transferred by air ambulance from Europe 4 days after a diving accident in which he sustained a $\mathrm{C} 5 / 6$ fracture dislocation resulting in complete $\mathrm{C} 5$ tetraplegia. He was intubated and ventilated and later tracheostomised following which he had an open reduction and internal fixation of his neck fracture. After weaning off 
the ventilator, the tracheostomy tube was exchanged for a minitracheotomy tube which was used for tracheal toilet and later removed.

\section{Discussion}

All the patients had complex respiratory problems with inability to expectorate sputum. This caused sputum retention and in Case 2, multiple episodes of lung collapse. We feel that without minitracheotomy, the first 3 patients would almost certainly have required bronchoscopy and/or intubation and ventilation perhaps followed by formal tracheostomy. It is well known that intubation and ventilation have their own complications (Bellamy et al., 1973; Whited et al., 1984; Hsu et al., 1987). Furthermore, Cheshire and Coats (1965) found higher mortality rates in patients undergoing early tracheostomies. They advocated fibre optic laryngoscopy for suction. Earlier, Cheshire and Foster (1964) had suggested endotracheal suction through a modified Magill tube. However, all these methods can cause patient exhaustion and are impractical for prolonged periods without ventilation.

Minitracheotomy (Matthews and Hopkinson, 1984) is a simple technique of introducing a small bore tube $(4 \mathrm{~mm})$ into the trachea through the cricothyroid membrane by a puncture incision under local anaesthetic. It can be carried out on the ward or ITU and greatly facilitates tracheal suction. It is now a well established technique since its introduction in 1981 (Preston et al., 1986).

\section{Advantages of minitracheotomy}

1. It is an easier procedure than tracheostomy and can be performed in the ward under local anaesthetic.

2. It is less exhausting for the patients and physiotherapist in clearing tracheal secretions, thus patients are able to cooperate better.

3. In addition to the minitracheotomy, patients can expectorate via the normal anatomical airway.

4. Patient's ability to talk is not impaired hence morale is not affected further.

5. Minitracheotomy may reduce the need for ventilation and formal tracheostomy.

6. The minitracheotomy tube can be left in situ for long periods with some care.

7. Tracheal stenosis has not been recorded.

8. The wound closes rapidly and the scar is small.

9. Patients are able to eat and drink normally.

10. Oxygenation and Nebulisation are possible through a mask.

11. It may be used as a weaning method from formal tracheostomy.

12. It avoids the complications of formal tracheostomy while allowing adequate tracheal suction.

13. It may reduce the need for bronchoscopic suction. 


\section{Disadvantages of minitracheotomy}

1. The suction technique is different from that used with an endotracheal tube or formal tracheostomy and requires adequate staff training.

2. Minitracheotomy tubes can be used to ventilate patients only for a very brief period. For continuous ventilation, a formal tracheostomy is required.

3. Complications such as haemorrhage and pneumothorax can occur at insertion.

\section{Conclusion}

The use of minitracheotomy in spinal injured patients has been described. We feel that it is a very useful technique for tracheal suction in certain cases. It avoids vigorous physiotherapy and is much less exhausting for the patient. Due to its small diameter it avoids the complications of traditional tracheostomy while providing adequate tracheal suction. However, skilled and meticulous suction techniques are necessary. While we feel that it reduces the need for bronchoscopic suction and probably also endotracheal intubation and tracheostomy, its role in the management of spinal injuries needs to be evaluated further. At the present time it should be regarded as an excellent method of tracheal suction and not as a replacement for tracheostomy and ventilation in the presence of indications for these procedures. Because of this, we believe that it is contra-indicated in patients whose respiratory failure may not be due to retained secretions alone, such as those with lesions above $\mathrm{C} 5$ or in patients with cervical lesions who show evidence of ascent of the neurological deficit. It is also contraindicated in children where the tracheal diameter is much smaller than adults.

\section{Acknowledgement}

We appreciate the help of Dr Thompson, Consultant Anaesthetist, Royal Shrewsbury Hospital for his assistance in teaching the minitracheotomy technique.

This work was not sponsored by the manufacturers of the minitracheotomy set.

\section{References}

Bellamy R, Pitts FW, StaufFer ES 1973 Respiratory complications in traumatic quadriplegia. fournal of Neurosurgery 39(5):596-600.

CARTER RE 1987 Respiratory Aspects of Spinal Cord Management. Paraplegia 25:262-266.

Cheshire DJE (1964 Respiratory management in acute traumatic tetraplegia. Paraplegia 1:252261.

CHeShIRE DJE, Foster KM 1964 An alternative technique for bronchial aspiration through a tracheostomy. Paraplegia 2:141-145.

Cheshire DJE, CoATs DA 1965 Respiratory and metabolic management in acute tetraplegia. Paraplegia 3:178-181.

Cheshire DJE 1966 Respiratory and Metabolic management in acute tetraplegia. Paraplegia 4:123.

DURBIN FC 1957 Fracture dislocation of the cervical spine. Fournal of Bone and foint Surgery 39B:23-38.

FORNER JV 1980 Lung volumes and mechanisms of breathing in tetraplegics. Paraplegia 18:258266.

GARDNER BP, WATT JWH, KRISHNAN KR 1986 The artificial ventilation of acute spinal cord damaged patients: A retrospective study of forty-four patients. Paraplegia 24:208-220. 
Gross D, LADD HW, RILEY EJ, et al. 1980 The Effect of training on strength and endurance of the diaphragm in quadriplegia. American fournal of Medicine 68:27-35.

Hsu S, DReisbach JN, Charlifue MA, et al. 1987 Glottic and tracheal stenosis in spinal cord injured patients. Paraplegia 25:136-148.

KIWERSKI J, WeISS M, ChrostowsKa T 1981 Analysis of mortality of patients after cervical spine trauma. Paraplegia 19:347-351.

MATTHEwS HR, Hopkinson RB 1984 Treatment of sputum retention by minitracheotomy. British fournal of Surgery 71:147-150.

PRESTON IM, MatThews HR, ReAdy AR 1986 Minitracheotomy: A new technique for tracheal suction. Physiotherapy 72(10):494-497.

SILVER JR, GIBBON NOK 1968 Prognosis in tetraplegia. British Medical fournal 2: 79-83.

TRIBE CR 1963 Causes of death in the early and late stages of paraplegia. Paraplegia 1: 19-46.

WHITED RE 1984 A prospective study of laryngotracheal sequelae in long-term intubation. Laryngoscope 94:367-377. 